\title{
EXISTENCE THEOREMS ACROSS A POINT OF RESONANCE
}

\author{
BY LAMBERTO CESARI
}

Communicated by Robert G. Bartle, April 8, 1976

1. Let us consider the equation

$$
E x+\alpha x=N x
$$

where $E: D(E) \rightarrow S, D(E) \subset S$, is a linear not necessarily bounded operator in a real Hilbert space $S$ with $1 \leqslant \operatorname{dim} W<\infty, W=\operatorname{ker} E$, and $N: S \rightarrow S$ is a continuous nonlinear operator. In terms of the alternative method (see Cesari [1], [2]), let $P: S \rightarrow S$ be the orthogonal projector with range $P S=S_{0}=W=\operatorname{ker} E$, let $S_{1}=(I-P) S$, and let $S_{1}$ be also the range of $E$. Let $H: S_{1} \rightarrow S_{1}$ denote a linear, bounded, compact operator (a partial inverse of $E$ ) such that the usual relations of the alternative method (selfadjoint case) hold: $\left(\mathrm{h}_{1}\right) H(I-P) E=I-P$; $\left(\mathrm{h}_{2}\right) E P=P E ;\left(\mathrm{h}_{3}\right) E H(I-P)=I-P$. Let $($,$) and \|\|$ denote inner product and norm in $S$, and let $L=\|H(I-P)\|$.

I (AN EXISTENCE THEOREM “AT RESONANCE”). Under the above hypotheses, let us assume that

(B) there is a constant $J_{0}>0$ such that $\|N x\| \leqslant J_{0}$ for all $x \in S$; and $\left(\mathrm{N}_{0}\right)$ there is a constant $R_{0} \geqslant 0$ such that $\left(N x, x^{*}\right) \leqslant 0$ [or always $\left.\left(N x, x^{*}\right) \geqslant 0\right]$ for all $x \in S, x^{*} \in S_{0}$ with $P x=x^{*},\left\|x^{*}\right\| \geqslant R_{0},\|x-P x\| \leqslant L J_{0}$.

Then the equation $E x=N x$ has at least a solution $x \in X$.

We refer to Cesari and Kannan [6] for a proof of I by Schauder's fixed point theorem, and to Kannan and McKenna [8] for a proof based on a Leray. Schauder argument. It has been pointed out that the hypotheses contained in recent specific and relevant theorems by Landesman and Lazer [9], Williams [13], Nečas [11] , and Lazer and Leach [10] concerning existence at resonance for boundary value problems for ordinary and partial differential equations, imply conditions (B) and $\left(\mathrm{N}_{0}\right)$. But the same hypotheses of the aformentioned specific theorems also imply the stronger condition $\left(\mathrm{N}_{\epsilon}\right)$ below. In turn, this condition has a stronger implication which we present here.

II (AN EXISTENCE THEOREM “ACROSS A POINT OF RESONANCE”). Under the same general hypotheses above, let us assume that (B) holds, and that

AMS (MOS) subject classifications (1970). Primary 47H15, 34B15, 34C15, 35G 30 , $35 \mathrm{~J} 40$. 
$\left(\mathrm{N}_{\epsilon}\right)$ there are constants $R_{0} \geqslant 0, \epsilon>0, K>L J_{0}$ such that $\left(N x, x^{*}\right) \leqslant$ $-\epsilon\left\|x^{*}\right\|$ (or always $\left.\left(N x, x^{*}\right) \geqslant \epsilon\left\|x^{*}\right\|\right)$ for all $x \in S, x^{*} \in S_{0}$ with $P x=x^{*},\left\|x^{*}\right\|$ $\geqslant R_{0},\|x-P x\| \leqslant K$.

Then, there are constants $\alpha_{0}>0, C>0$ such that, for every $|\alpha| \leqslant \alpha_{0}$, the equation $E x+\alpha x=N x$ has at least a solution $x$ with $\|x\| \leqslant C$.

We refer to Cesari [3] for a proof of II by Schauder's fixed point theorem. Note that, in equation (1), the parameter $\alpha$ can "move across the point of resonance $\alpha=0$ ", without the solution becoming infinite, due to the nonlinearity of the problem. Our result II, in the large and under sole qualitative hypotheses, seems to be new and of some physical significance. In [3], [4], [5] Cesari has also proved more general statements, with $S$ replaced by Banach spaces $X, Y$, for $N: X \rightarrow Y$ continuous and of slow growth, and the term $\alpha x$ replaced by $\alpha A x$, with $A: X \rightarrow Y$ any continuous, nonlinear operator, mapping bounded sets into bounded sets. As applications of II we restate, for instance, the theorems of Landesman and Lazer, Williams, and Lazer and Leach, in a stronger form.

2. Let us consider the ordinary differential equation

$$
x^{\prime \prime}+\left(m^{2}+\alpha\right) x+h(x)=p(t), \quad x \text { scalar, }
$$

where $m$ is an integer, $h$ is a real valued function in $(-\infty,+\infty), p(t)$ is a $2 \pi$ periodic function, and $\alpha$ is a real parameter.

III. If $p$ is continuous and $2 \pi$-periodic, if $h$ is continuous with $|h(s)| \leqslant M$ for all real $s$, and if there are constants $c<d, C<D$ such that $h(s) \leqslant C$ for $s \leqslant c ; h(s) \geqslant D$ for $s \geqslant d$, and moreover $\left(A^{2}+B^{2}\right)^{1 / 2}<2(D-C)$, where $A=$ $\int_{0}^{2 \pi} p(t) \cos m t d t, B=\int_{0}^{2 \pi} p(t) \sin m t d t$, then there are constants $\alpha_{0}>0, M>0$ such that for every $|\alpha| \leqslant \alpha_{0}$, equation (2) has at least a $2 \pi$-periodic solution $x(t)$, $-\infty<t<+\infty$, with $|x(t)| \leqslant M$.

For $\alpha=0$ this theorem was proved by Lazer and Leach [8]. The same statement holds also for $h$ replaced by $-h$.

3. Let us consider the partial differential equation

$$
E x+\alpha x+h(x)=f(t), \quad t \in G,
$$

where $G$ is a bounded domain in $R^{\nu}$ of points $t=\left(t_{1}, \ldots, t_{\nu}\right), E$ is the differential operator of order $2 m$ in $G$ defined by

$$
E x=\sum_{|\alpha|,|\beta| \leqslant m}(-1)^{|\beta|} D^{\beta}\left(a_{\alpha \beta}(t) D^{\alpha} x\right), \quad t \in G,
$$

with $a_{\alpha \beta}=a_{\beta \alpha}$, and $\Sigma a_{\alpha \beta}(t) \zeta^{\alpha} \zeta^{\beta} \geqslant c|\zeta|^{2 m}$ for some constant $c>0$ and where $\Sigma$ ranges over all $|\alpha|=|\beta|=m$. In order to simplify the exposition, and follow- 
ing de Figueiredo, we limit ourselves to strong solutions, and therefore under some smoothness hypotheses, say $a_{\alpha \beta} \in C^{|\beta|}$ in $\bar{G}$, and $\partial G$ of class $C^{2 m}$. We assume that the homogeneous linear Dirichlet problem $E x=0, x \in H_{0}^{m} \cap H^{2 m}$, has nontrivial solutions, thus, $1 \leqslant \operatorname{dim} W<\infty, W=\operatorname{ker} E$. Let $h(s),-\infty<s<$ $+\infty$, be a continuous real valued function, for which the limits $R=h(+\infty)$ and $r=h(-\infty)$ exist and are finite. For every element $w \in W$ let $G^{+}, G^{-}$denote the subsets of all $t \in G$, where $w(t) \geqslant 0$ and $w(t) \leqslant 0$, respectively, and let $w^{+}=$ $\int_{G}+|w| d t, w^{-}=\int_{G^{-}}|w| d t$.

IV. Under the conditions above and for $f \in L_{2}(G)$, if for every $w \in W$ we also have $R w^{-}-r w^{+}<\int_{G} f(t) w(t) d t<R w^{+}-r w^{-}$, then there are constants $\alpha_{0}>0, C>0$ such that, for every real $\alpha$ with $|\alpha| \leqslant \alpha_{0}$, equation (3) has at least a solution $x \in H_{\theta}^{m} \cap H^{2 m}$ with $\|x\|_{2 m} \leqslant C$.

The same statement holds also for $h$ replaced by $-h$. For $\alpha=0, m=1$ this statement was proved by Landesman and Lazer [9], and for $\alpha=0, m \geqslant 1$ by Williams [13]. The case in which $h$ depends on $t, x$, and the partial derivatives of $x$ of orders $\leqslant 2 m-1$ has been studied by de Figueiredo [7] for $\alpha=0$, and his statement too can be restated in the strong form above. Analogous results have been proved for the case in which $h$ may grow with $x$ of order $r, 0 \leqslant$ $r<1$, by de Figueiredo [7] for $\alpha=0$, and these statements too can be given the strong form above in terms of the alternative method (cf. Cesari [4]). For nonselfadjoint problems, see Shaw [12] and Cesari [4], [5].

\section{REFERENCES}

1. L. Cesari, Functional analysis and periodic solutions of nonlinear differential equations, Contributions to Differential Equations 1 (1963), 149-187. MR 27 \#1662.

2. - Alternative methods in nonlinear analysis, Proc. Internat. Conf. Differential Equations (Univ. of Southern California, Los Angeles, 1974), Academic Press, New York, 1975, pp. 95-148.

3. - An abstract existence theorem across a point of resonance, Proc. Internat. Sympos. Dynamical Systems (Univ. of Florida, Gainesville, March 1976), Academic Press, New York (to appear).

4. Nonlinear oscillations across a point of resonance for nonselfadjoint systems, J. Differential Equations (to appear).

5. Nonlinear problems across a point of resonance for nonselfadjoint systems, Nonlinear Analysis, Academic Press (to appear).

6. L. Cesari and R. Kannan, An abstract existence theorem at resonance, Proc. Amer. Math. Soc. (to appear).

7. D. G. de Figueiredo, The Dirichlet problem for nonlinear elliptic equations, a Hilbert space approach, Lectures Notes in Math., vol. 446, Springer-Verlag, Berlin and New York, 1975, pp. 144-165.

8. R. Kannan and P. J. McKenna, An existence theorem by alternative methods for semilinear abstract equations, Boll. Un. Mat. Ital. (to appear).

9. E. M. Landesman and A. C. Lazer, Nonlinear perturbations of linear elliptic boundary value problems at resonance, J. Math. Mech. 19 (1969/70), 609-623. MR 42 \#2171. 
10. A. C. Lazer and D. E. Leach, Bounded perturbations of forced harmonic oscillators at resonance, Ann. Mat. Pura Appl. (4) 82 (1969), 49-68. MR 40 \#2972.

11. J. Nečas, The range of nonlinear operators with linear asymptotes which are not invertible, Comment. Math. Univ. Carolinae 14 (1973), 63-72. MR 47 \#741.

12. H. C. Shaw, Nonlinear elliptic boundary value problems at resonance, J. Differential Equations (to appear).

13. S. A. Williams, $A$ sharp sufficient condition for solution of a nonlinear elliptic boundary value problem, J Differential Equations 8 (1970), 580-586. MR 42 \#2169.

DEPARTMENT OF MATHEMATICS, UNIVERSITY OF MICHIGAN, ANN ARBOR, MICHIGAN 48109 